\title{
Clinical investigation of EGFR mutation detection by pyrosequencing in lung cancer patients
}

\author{
HEE JOUNG KIM ${ }^{1}$, SEO YOUNG OH ${ }^{2}$, WAN SEOP KIM ${ }^{2}$, SUN JONG KIM ${ }^{1}$, \\ GWANG HA YOO ${ }^{1}$, WON DONG KIM ${ }^{1}$ and KYE YOUNG LEE ${ }^{1}$
}

Departments of ${ }^{1}$ Internal Medicine and ${ }^{2}$ Pathology, Konkuk University School of Medicine, Seoul 143-729, Republic of Korea

Received July 10, 2012; Accepted September 24, 2012

DOI: 10.3892/ol.2012.950

\begin{abstract}
Direct sequencing is the standard method for the detection of epidermal growth factor receptor (EGFR) mutations in lung cancer, however, its relatively low sensitivity limits its clinical use. Pyrosequencing is a bioluminometric, real-time non-electrophoretic DNA sequencing technique with a number of advantages compared with direct sequencing, including higher sensitivity, speed, automation and costeffectiveness. Clinical specimens from 202 lung cancer patients were analyzed for EGFR mutations in exons 18, 19, 20 and 21 using the pyrosequencing method following genomic DNA extraction from paraffin-embedded tissue specimens. All clinical data and tumor specimens were obtained from the Konkuk University Hospital (Korea) between July 2006 and December 2008. The results and clinical responses to EGFR-tyrosine kinase inhibitors (TKIs) were compared. Overall, EGFR mutation-positive rate was $26.7 \%$ (54/202). Activating EGFR mutations were observed more frequently in female (52.1 vs. $13.0 \%$ ), non-smoking (47.8 vs. $15.8 \%$ ) and adenocarcinoma (35.2 vs. $5.2 \%$ ) patients. However, significant numbers of EGFR mutation-positive patients were identified as male, former or current smokers and non-adenocarcinoma patients. The combinations of favorable clinicopathological factors, including female, non-smoking and adenocarcinoma, were not identified to significantly increase the positive EGFR mutation rate (female, 52.1\%; female and non-smoker, 52.6\%; female, non-smoker and adenocarcinoma, 51.9\%). The present findings indicate that EGFR mutation analysis is a highly useful method for the prediction of response to EGFR-TKI and the use of favorable clinicopathological factors to perform this analysis is not suitable. Exon 19 deletion was the most common mutation (63.6\%) and exon 21 L858R substitution was measured at $32.7 \%$. The exon 20 T790M mutation was identified in 1 patient prior to EGFR-TKI treatment. EGFR
\end{abstract}

Correspondence to: Professor Kye Young Lee, Department of Internal Medicine, Konkuk University School of Medicine, 120-1 Neungdong-ro, Hwayang-dong, Gwangjin-gu, Seoul, Republic of Korea

E-mail: kyleemd@kuh.ac.kr

Key words: EGFR mutation, pyrosequencing, lung cancer mutation status is associated with response to EGFR-TKI and the overall response rate in patients who have the activating EGFR mutation was 82.4 vs. $5.9 \%$ in patients with a wild-type EGFR. The present study demonstrates that EGFR mutations analyzed by the pyrosequencing method are well correlated with clinicopathological parameters and that this method may be useful in the clinical practice.

\section{Introduction}

Previous studies have demonstrated that epidermal growth factor receptor tyrosine kinase inhibitors (EGFR-TKIs), including gefitinib and erlotinib, are more effective as the first-line of treatment compared with platinum-based cytotoxic chemotherapy (1-4) for patients with advanced non-small cell lung cancer (NSCLC) and classical activating EGFR gene mutations, including exon 19 deletion and exon 21 point mutation (L858R). Based on an excellent response to EGFR-TKIs in NSCLC patients with EGFR mutations, gefitinib was approved in April 2011 as the first-line of treatment for NSCLC patients with activating EGFR mutations by the Health Insurance Review and Assessment Service in South Korea. However, the consensus for EGFR mutation testing in NSCLC, including which case to select and when to test, is not well established, particularly with respect to technical methods of EGFR mutation detection. A variety of methods, including direct sequencing, amplification refractory mutation system, length analysis, denaturing high-performance liquid chromatography and peptide nucleic acid-mediated real-time polymerase chain reaction (PCR) clamping (5-11), have been proposed as suitable methods for the detection of EGFR mutations. Among these various assays, direct sequencing of amplified DNA products is widely used and is considered the standard method. To identify novel mutations, direct sequencing must be adopted as this method identifies exact mutations without sample batch processing. However, direct sequencing involves multiple steps, including DNA extraction, PCR-based amplification, DNA sequencing and sequence interpretation, typically requiring several days to obtain a result following tissue acquisition. In addition, suboptimal mutation detection sensitivity in clinical tumor samples is a major disadvantage of direct sequencing and currently total DNA samples must be composed of more than $25 \%$ mutant DNA for mutation detection (12). 
Pyrosequencing is a DNA sequencing technology based on the sequencing-by-synthesis principle $(13,14)$. This technique is a real-time bioluminescence technique in which the phosphate released during the incorporation of a nucleotide into a growing DNA chain is converted into light through a series of enzymatic reactions. Pyrosequencing identifies individual bases or short stretches of nucleic acid sequence at predetermined positions. The technique is a simple, robust, fast and sensitive method that is also cost-effective. There are a number of studies that have applied the pyrosequencing technique to analyze genetic variations, including k-ras and BRAF (15-17). However, there are currently no studies on the application of pyrosequencing for the detection of EGFR mutations in clinical practice.

In the present study, we analyzed 202 lung cancer patients for EGFR mutations detected by pyrosequencing in a single-center, prospective study. We also analyzed the clinicopathological parameters and correlations between EGFR genotype and response to EGFR-TKIs, including gefitinib and erlotinib.

\section{Materials and methods}

Patient population. Patients included in the study were pathologically confirmed to have lung cancer at Konkuk University Hospital (Seoul, South Korea) between July 2006 and December 2008. Patients were prospectively observed for tumor response and survival outcome. The present study was reviewed and approved by the Institutional Ethic Committee at Konkuk University Hospital. All patients provided informed consent to participate in the study and permission for use of tumor tissues.

DNA extraction. Areas of the tissue and cytology slides containing the tumor cells of interest were marked by a cytopathologist using a pen. A diamond-tipped pencil was then used to mark the underside of the slide. Following removal of the cover slip, tumor cells were scraped with a 26-gauge needle. Briefly, 50-100 $\mu 1$ of DNA extraction buffer solution $(50 \mathrm{mM}$ Tris buffer, $\mathrm{pH} 8.3 ; 1 \mathrm{mM}$ EDTA, $\mathrm{pH}$ 8.0; 5\% Tween-20 and $200 \mu \mathrm{g} / \mathrm{ml}$ proteinase $\mathrm{K}$ ) with $10 \%$ resin was added to the scraped cells. Following incubation at $56^{\circ} \mathrm{C}$ for at least $1 \mathrm{~h}$, the tube was heated to $100^{\circ} \mathrm{C}$ for $20 \mathrm{~min}$ followed by centrifugation at $12,000 \mathrm{rpm}$ for $10 \mathrm{~min}$ at $4^{\circ} \mathrm{C}$ to pellet the debris. The recovered supernatant was used for the PCR.

EGFR mutation analysis using pyrosequencing. Biotinylated (B) PCR primer sequences for the amplification of EGFR mutation sites were as follows: exon 18, 5'-B-GCTCCC AACCAAGCTCTCTT-3'(F) and 5'-TATACACCGTGCCGA ACGC-3'(R); exon 19, 5'-GCATGTGGCACCATCTCA-3'(F) and 5'-B-AAAAGGTGGGCCTGAGGTT-3'(R); exon 20, 5'-B-ATGGCCAGCGTGGACAAC-3'(F) and 5'-TTTGTG TTCCCGGACATAGTC-3'(R); exon 21, 5'- ACCGCAGCA TGTCAAGATCAC-3'(F) and 5'-B-TCCGCACCCAGC AGTTTG-3'(R). In this method, $5 \mu 1$ of DNA was added to produce a $50 \mu 1$ of PCR solution mixture that contained $0.2 \mathrm{mmol}$ of each dNTP, $1.5 \mathrm{mmol} / 1 \mathrm{MgCl}_{2}$, 1 X PCR buffer, 1.5 units of immolase DNA Taq polymerase and 20 pmol of each primer. PCR was performed with an initial denaturation for $5 \mathrm{~min}$ at $95^{\circ} \mathrm{C}$ followed by 40 cycles of $30 \mathrm{sec}$ at $95^{\circ} \mathrm{C}$, $30 \mathrm{sec}$ at $54^{\circ} \mathrm{C}$ (exons 18,21$), 60^{\circ} \mathrm{C}$ (exon 19) or $55^{\circ} \mathrm{C}$ (exon 21 ), $30 \mathrm{sec}$ at $72^{\circ} \mathrm{C}$ and incubation for $10 \mathrm{~min}$ at $72^{\circ} \mathrm{C}$. PCR products were resolved by agarose gel electrophoresis to confirm successful amplification. The biotinylated products were then immobilized to streptavidin-coated beads using a solution from a commercial PSQTM96 sample preparation kit. Beads (3 $\mu \mathrm{l}$ ) were diluted in binding buffer with $10 \mu \mathrm{l}$ biotinylated PCR products, incubated for $10 \mathrm{~min}$ at room temperature and then transferred to a filter probe where the liquid was removed by vacuum filtration. DNA in the denaturation solution was separated; the templates were washed with washing buffer, transferred to a PSQ 96 SNP plate and annealed with the following sequencing primers: exon 18 E709K, 5'TGATCTTTTTGAATTCAGTT-3' and G719A, 5'-CCGAAC GCACCGGAG-3'; exon 19 deletion, 5'-ATTCCCGTC GCTATC-3'; exon 20 T790M, 5'-GATGCCCAGCAGGCG-3'; and exon 21 L858R and A859T, 5'-AAGATCACAGAT TTTGG-3' in annealing buffer at room temperature. Finally, the samples were analyzed using a PyroMark ID System and SNP reagent kit (both purchased from Biotage, Uppsala, Sweden).

Statistical analysis. The association between the EGFR mutational status and clinical characteristics, including gender, smoking status, stage, tumor specimen, pathological subtype and clinical response to EGFR-TKI, was assessed using the $\chi^{2}$ test. The age between the two groups was analyzed by the Mann-Whitney U test. Using binary logistic regression, the EGFR mutations, according to gender, pathological subtype and smoking status, were evaluated to investigate the effect of each variable on the development of the EGFR mutations. All analyses were performed using SPSS version 17.0 (SPSS Inc., Chicago, IL, USA). $\mathrm{P}<0.05$ was considered to indicate a statistically significant difference.

\section{Results}

Patient characteristics. In total, 202 tumor samples were tested for EGFR mutations in exons 18, 19, 20 and 21 (Table I). The median age of the enrolled patients was 64 years (range, $25-87$ years). Overall, 71 of the patients were female (35.1\%) and 69 were non-smokers (34.2\%). The majority of the histological diagnoses examined were adenocarcinomas (71.8\%), followed by squamous cell carcinomas (SCCs; 19.3\%; Table II). EGFR mutations were more frequent in female $(68.5 \%)$, non-smoking $(61.1 \%)$ and adenocarcinoma patients (94.4\%). Unexpectedly, cytological were superior to biopsy specimens in determining the EGFR mutation status ( 45.2 vs. $21.9 \%, \mathrm{P}=0.02$ ), indicating that a few well-selected malignant cells were sufficient to analyze EGFR mutations.

EGFR mutations in tumor tissue DNA. Mutations were identified in $54(26.7 \%)$ of the 202 patients by pyrosequencing (Table III). Of the 55 mutations, the most common mutations were in-frame deletions at exon 19 and an arginine-for-leucine substitution at amino acid 858 (L858R); the frequencies of these mutations were $35(63.6 \%)$ and 17 (30.9\%), respectively. Each of the following mutations was identified once; glycinefor-alanine substitution at amino acid 719 (G719A) in exon 18, 
Table I. EGFR mutation data using pyrosequencing $(n=202)$.

\begin{tabular}{|c|c|c|c|c|}
\hline \multirow[b]{2}{*}{ Characteristics } & \multicolumn{2}{|c|}{ EGFR genotype } & \multirow[b]{2}{*}{ Total } & \multirow[b]{2}{*}{ P-value } \\
\hline & Mutant & Wild-type & & \\
\hline Patients, n (\%) & $54(26.7)$ & $148(73.3)$ & 202 & \\
\hline Age (years), mean $\pm \mathrm{SD}$ & $65.2 \pm 10.5$ & $64.0 \pm 11.6$ & $64.3 \pm 11.3$ & 0.495 \\
\hline Median (range) & $66(41-83)$ & $66(25-87)$ & $66(25-87)$ & \\
\hline Gender, n (\%) & & & & $<0.001$ \\
\hline Male & $17(13.0)$ & $114(87.0)$ & 131 & \\
\hline Female & $37(52.1)$ & $34(47.9)$ & 71 & \\
\hline Smoking status & & & & $<0.001$ \\
\hline Non, n (\%) & $33(47.8)$ & $36(52.2)$ & 69 & \\
\hline $\mathrm{Ex}, \mathrm{n}(\%)$ & $12(18.2)$ & $54(81.8)$ & 66 & \\
\hline Current, n (\%) & $9(13.4)$ & $58(86.6)$ & 67 & \\
\hline Pack-years, mean \pm SD & $36.4 \pm 22.5$ & $37.6 \pm 17.7$ & $37.5 \pm 18.4$ & \\
\hline Stage, n (\%) & & & & 0.073 \\
\hline I & $5(27.8)$ & $13(72.2)$ & 18 & \\
\hline II & $0(0.0)$ & $7(100)$ & 7 & \\
\hline III & $15(20.3)$ & $59(79.7)$ & 74 & \\
\hline IV & $34(34.3)$ & $65(65.7)$ & 99 & \\
\hline M-stage, n (\%) & & & & 0.021 \\
\hline 0 & $20(19.6)$ & $82(80.4)$ & 102 & \\
\hline 1 & $34(34.0)$ & $66(66.0)$ & 100 & \\
\hline Diagnostic tool, n (\%) & & & & 0.02 \\
\hline Cytology & $19(45.2)$ & $23(54.8)$ & 42 & \\
\hline Biopsy & $35(21.9)$ & $125(78.1)$ & 160 & \\
\hline
\end{tabular}

EGFR, epidermal growth factor receptor.

Table II. Pathological correlation with EGFR mutation status.

\begin{tabular}{lccc}
\hline & \multicolumn{2}{c}{ EGFR genotype (\%) } & \\
\cline { 2 - 3 } Variable & Mutant & Wild-type & Total \\
\hline Patients & $54(26.7)$ & $148(73.3)$ & 202 \\
Adenoca & $45(34.1)$ & $87(65.9)$ & 132 \\
Adenoca & & & \\
+ BAC & $5(62.5)$ & $3(37.5)$ & 8 \\
BAC & $2(50.0)$ & $2(50.0)$ & 4 \\
SqCC & $2(5.1)$ & $37(94.9)$ & 39 \\
Large-cell & $0(0.0)$ & $8(100)$ & 8 \\
carcinoma & $1(16.7)$ & $5(83.3)$ & 6 \\
NSCLC & $0(0.0)$ & $4(100)$ & 4 \\
SCLC & & & \\
Double primary & $1(33.3)$ & $2(66.7)$ & 3 \\
(Adenoca + SqCC) & & & \\
Total & & & \\
Adenoca & $51(35.2)$ & $94(64.8)$ & $145(71.8)$ \\
Non-Adenoca & $3(5.2)$ & $54(93.1)$ & $58(28.7)$ \\
\hline
\end{tabular}

EGFR, epidermal growth factor receptor. Adenoca, adenocarcinoma; BAC, bronchioloalveolar carcinoma; SqCC, squamous cell carcinoma; SCLC small cell lung cancer, NSCLC, non-SCLC. threonine-for-methionine substitution at amino acid 790 (T790M) in exon 20 and alanine-for-threonine substitution at amino acid 859 (A859T) in exon 21. Notably, one patient had in-frame deletions in exon 19 and T790M in exon 20. However, the patient did not receive EGFR-TKI treatment and therefore response to targeted drug treatment was not evaluated.

Correlation between EGFR mutations and clinicopathological parameters. The positive predictive value (PPV) of each clinical factor was analyzed (Table IV). Among 71 females, mutations were detected in 37 patients $(52.1 \%)$ and the PPVs of the non-smoker and adenocarcinoma patients were $47.8 \%$ (33/69) and 40.8\% (51/125), respectively. Although EGFR mutations are commonly detected in female patients with adenocarcinoma who had never smoked, an accurate estimate of the mutation status solely from clinicopathological parameters was not obtained. The proportions of patients who had one, two or three favorable clinicopathological factors out of 54 patients who had an EGFR mutation were $27.8(15 / 54)$, $20.4(11 / 54)$ and $51.9 \%$ (28/54), respectively. No male patients with a non-adenocarcinoma histology who previously smoked had an EGFR mutation.

Binary logistic regression analyses were conducted to predict the EGFR criteria according to gender, pathological 
Table III. EGFR mutation profile.

\begin{tabular}{lclr}
\hline Exon and mutation profile & Patients, $\mathrm{n}(\%)$ & Mutation & Patients, $\mathrm{n}(\%)$ \\
\hline 18 & $1(1.8)$ & G719A & 1 \\
19 & $35(63.6)$ & Deletion & Deletion and point mutation \\
& & Point mutation & L858R \\
& & & A859T \\
21 & $18(32.7)$ & T790M and L858R & 17 \\
20 and 21 & & & 1 \\
\hline
\end{tabular}

EGFR, epidermal growth factor receptor. $n=54$.

Table IV. Clinicopathological correlation with EGFR mutation.

\begin{tabular}{lc}
\hline Clinicopathological factor & EGFR mutation/total $(\%)$ \\
\hline Female & $37 / 71(52.1)$ \\
Non-smoker & $33 / 69(47.8)$ \\
Adenoca & $51 / 125(35.2)$ \\
Female and non-smoker & $30 / 57(52.6)$ \\
Female and adenoca & $35 / 65(53.8)$ \\
Non-smoker and adenoca & $30 / 60(47.6)$ \\
Female, non-smoker and adenoca & $28 / 54(51.9)$ \\
\hline
\end{tabular}

EGFR, epidermal growth factor receptor; adenoca, adenocarcinoma.

subtype (i.e., adenocarcinoma) and smoking status. The results are presented in Table V. Gender and pathological subtype were important variables with respect to the EGFR mutations. Gender was identified to be significantly correlated with EGFR genotype $(\mathrm{P}=0.002)$ and the odds ratios indicated that females had an increased probability of having EGFR mutations. Similarly, a significant correlation was identified between histological results and mutation status $(\mathrm{P}=0.007)$. Patients with adenocarcinoma was more closely associated with mutation status compared with other pathologies. However, by logistic analyses, smoking status was not identified to have a significant effect on EGFR mutation status in the samples. The Wald value (0.17) indicated an insignificant difference of 0.683 , which revealed that non-smoking was unlikely to be directly associated with mutation status.

Correlation between EGFR genotype and response to EGFR-TKI. Of 202 patients who were tested for EGFR mutations, 92 patients were treated with EGFR-TKIs in their treatment course. Among 38 patients with positive mutations detected by pyrosequencing, $2(5.3 \%)$ had a complete response, $26(68.4 \%)$ had a partial response and $4(10.5 \%)$ had stable disease. The best patient responses to EGFR-TKI treatment yielded a total disease control rate of 94.1\% (Table VI). Mutation-positive patients demonstrated a more favorable prognosis and longer progression-free survival (mutation-positive vs. negative patients, 9.2 vs. 2.6 months; $\mathrm{P}<0.001$ ). This observation was as expected. The present results indicate that EGFR mutation status identified by pyrosequencing is well correlated with the response to EGFR-TKI treatment of lung cancer patients.

\section{Discussion}

In 2010, the National Comprehensive Cancer Network practice guidelines recommended that targeted therapies should be used as the first line of treatment against NSCLC patients who have the EGFR activating mutation. This recommendation was based on a number of clinical studies, performed following the initial identification of specific mutations in the EGFR tyrosine kinase domain in 2004 (15,16), including a 2009 IPASS report $(1,2,18,19)$. Previously, EGFR mutation testing was recommended prior to systemic chemotherapy for all patients with advanced NSCLC excluding SCC (20). To select the appropriate treatment regimen, rapid and accurate mutation test results are necessary in clinical practice. Although various methods are used to detect EGFR mutations, there is no universal consensus on which method is most effective. Direct DNA sequencing is the current gold standard for EGFR mutation detection, however, this method is labor-intensive, time-consuming and has a low level of sensitivity (21).

Pyrosequencing is a nonelectrophoretic nucleotide extension sequencing method that is performed for various applications (22), including SNP genotyping, mutation detection in tumors (23) and quantitative $\mathrm{CpG}$ island methylation analyses $(24,25)$. In the present study, we evaluated a sensitive sequencing assay for the detection of EGFR mutations using pyrosequencing technology. We demonstrate that pyrosequencing results reveal a good predictive value for clinical responses to EGFR-TKI treatment and detection of the mutation in mutant/wild-type mixed DNA samples derived from paraffin-embedded tissue is a sensitive, rapid and simple method to perform. However, this method is not suitable for the detection of novel mutations.

EGFR mutations were detected in 54 (26.7\%) of 202 patients with NSCLC and 51 (35.2\%) of 145 adenocarcinoma patients. In patients with SCC, the observed incidence was $5.1 \%$ (2/39). Forbes et al previously stated that this EGFR mutation frequency does not justify routine testing 
Table V. Odds ratios for EGFR mutations by gender, pathological type and smoking status.

\begin{tabular}{lcllccc}
\hline Status & B & S.E. & Wald & Significance & Exp (B) & $95 \%$ CI \\
\hline Gender & -1.53 & 0.050 & 9.38 & 0.002 & 0.216 & $0.081-0.576$ \\
Pathology & -1.73 & 0.64 & 7.29 & 0.007 & 0.177 & $0.050-0.622$ \\
Smoking & 0.21 & 0.50 & 0.17 & 0.683 & 1.227 & $0.459-3.278$ \\
\hline
\end{tabular}

EGFR, epidermal growth factor receptor. B, B coefficient; S.E., standard error; Exp, exponentiation; CI, confidence interval.

Table VI. Clinical response to EGFR-TKI treatment according to EGFR genotype.

\begin{tabular}{|c|c|c|c|c|}
\hline & \multicolumn{2}{|c|}{ EGFR genotype } & \multirow[b]{2}{*}{ Total } & \multirow[b]{2}{*}{ P-value } \\
\hline & Mutant & Wild-type & & \\
\hline Patient, $\mathrm{n}$ & 38 & 54 & 92 & \\
\hline Response evaluation, $\mathrm{n}(\%)$ & & & & $<0.001$ \\
\hline $\mathrm{CR}$ & $2(5.3)$ & $0(0)$ & 2 & \\
\hline PR & $26(68.4)$ & $3(5.6)$ & 29 & \\
\hline $\mathrm{SD}$ & $4(10.5)$ & $7(13.0)$ & 11 & \\
\hline $\mathrm{PD}$ & $2(5.3)$ & $41(75.9)$ & 43 & \\
\hline ND & $4(10.5)$ & $3(5.6)$ & 7 & \\
\hline ORR (CR+PR), n/total (\%) & $28 / 34(82.4)$ & $3 / 51(5.9)$ & $31 / 85(36.5)$ & $<0.001$ \\
\hline DCR $(\mathrm{CR}+\mathrm{PR}+\mathrm{SD}), \mathrm{n} /$ total $(\%)$ & $32 / 34(94.1)$ & $10 / 51(19.6)$ & $42 / 85(49.4)$ & $<0.001$ \\
\hline Duration of TKI (months), mean \pm SD & $9.2 \pm 5.1$ & $2.6 \pm 3.4$ & $5.3 \pm 5.3$ & $<0.001$ \\
\hline
\end{tabular}

EGFR, epidermal growth factor receptor. CR, complete response; PR, partial response; SD, stable disease; PD, progressive disease; ND, not determined; ORR, objective response rate; DCR, disease control rate; TKI, tyrosine kinase inhibitor.

of all tumor specimens (26). Similar observations were identified in small cell lung cancer cases where no mutation was observed in the 4 patients. In these cases, meticulous pathological evaluation is necessary to precisely classify the histological type of lung cancer. Analysis of 42 cytological specimens revealed 19 mutations. In this case the pyrosequencing method was sufficiently sensitive to detect EGFR mutations using a small number of well-selected malignant cells, as suggested by Rekhtman et al (27). Using cytology specimens to test for EGFR mutations instead of performing an additional biopsy may prove an excellent follow-up tool to determine if additional resistant mutations, including T790M, have developed.

In the present study, we evaluated the utility of pyrosequencing to assess the EGFR mutation status in patients with lung cancer in a diagnostic setting. Median progression-free survival to EGFR-TKI treatment in patients with an activating EGFR mutation was 9.2 months, consistent with data from a randomized controlled trial $(12,19)$. In addition, the mutation results correlated well with the clinical course. PPVs of the good clinical factors did not increase in proportion to the numbers of favorable clinical factors identified in patients. Even in patients with all favorable clinical factors, including female, adenocarcinoma, non-smoker and Asian ethnicity, a wild-type EGFR mutation was identifed in more than one-third. These results support the hypothesis that EGFR mutation testing is required in all patients diagnosed with NSCLC, excluding SCC.

As predicted, a marked association between gender and histological subtype by EGFR mutation was observed. A significant correlation between females with adenocarcinoma and identification of an EGFR mutation was revealed and this observation was consistent with previous findings $(28,29)$. By contrast, analysis of the smoking status variable produced notable findings in the present study and we did not identify a significant correlation between smoking status and EGFR mutations. The reason for these inconsistancies is currently unknown, however, it may be associated with histological heterogeneity. Additional factors that may contribute to this difference include inaccurate recall of smoking history and passive smokers defined as non-smokers. Nevertheless, we should not overlook the possibility that male patients who are former or current smokers may have EGFR mutations.

The present data demonstrate the importance of EGFR mutation analysis in clinical treatment of lung cancer and the pyrosequencing method is a sensitive and valuable methodology for analyzing EGFR mutations.

\section{Acknowledgements}

The present study was supported by a grant-in-aid from Konkuk University in 2009. 


\section{References}

1. Mok TS, Wu YL, Thongprasert S, et al: Gefitinib or carboplatinpaclitaxel in pulmonary adenocarcinoma. N Engl J Med 361: 947-957, 2009.

2. Mitsudomi T, Morita S, Yatabe Y, et al: Gefitinib versus cisplatin plus docetaxel in patients with non-small-cell lung cancer harbouring mutations of the epidermal growth factor receptor (WJTOG3405): open label, randomized phase 3 trial. Lancet Oncol 11: 121-128, 2010.

3. Sequist LV, Martins RG, Spigel D, et al: First-line gefitinib in patients with advanced non-small cell lung cancer harboring somatic EGFR mutations. J Clin Oncol 26: 2442-2449, 2008.

4. Rosell R, Viteri S, Molina MA, et al: Epidermal growth factor receptor tyrosine kinase inhibitors as first-line treatment in advanced nonsmall-cell lung cancer. Curr Opin Oncol 22: $112-120,2010$.

5. Kimura H, Kasahara K, Kawaishi M, et al: Detection of epidermal growth factor receptor mutations in serum as a predictor of the response to gefitinib in patients with non-small cell lung cancer. Clin Cancer Res 12: 3915-3921, 2006.

6. Kimura H, Fujiwara Y, Sone T, et al: High sensitivity detection of epidermal growth factor receptor mutations in the pleural effusion of non-small cell lung cancer patients. Cancer Sci 97 642-648, 2006

7. Molina-Vila MA, Bertran-Alamillo J, Reguart N, et al: A sensitive method for detecting EGFR mutations in non-smal cell lung cancer samples with few tumor cells. J Thorac Oncol 3: 1224-1235, 2008.

8. Cohen V, Agulnik JS, Ang C, et al: Epidermal growth factor receptor mutations detected by denaturing high-performance liquid chromatography in nonsmall cell lung cancer. Cancer 111: 4309-4317, 2010.

9. Kim HJ, Lee KY, Kim YC, et al: Detection and comparison of peptide nucleic acid-mediated real-time polymerase chain reaction clamping and direct gene sequencing for epidermal growth factor receptor mutations in patients with non-small cell lung cancer. Lung Cancer 75: 321-325, 2012.

10. Lee KY, Kim HJ, Kim SJ, et al: PNA-Mediated PCR Clamping for the detection of EGFR mutations in non-small cell lung cancer. Tuberc Respir Dis 69: 271-278, 2010.

11. Kim HJ, Kim WS, Shin KC, et al: Comparative analysis of peptide nucleic acid (PNA)-mediated real-time PCR clamping and DNA direct sequencing for EGFR mutation detection. Tuberc Respir Dis 70: 21-27, 2011.

12. Pao W and Ladanyi $\mathrm{M}$ : Epidermal growth factor receptor mutation testing in lung cancer, searching for the ideal method. Clin Cancer Res 13: 4954-4955, 2007.

13. Langaee $\mathrm{T}$ and Ronaghi M: Genetic variation analyses by pyrosequencing. Mutat Res 573: 96-102, 2005.
14. Ahmadian A, Ehn M and Hober S: Pyrosequencing: history, biochemistry and future. Clin Chim Acta 363: 83-94, 2006.

15. Dufort S, Richard MJ and Fraipont F: Pyrosequencing method to detect KRAS mutation in formalin-fixed and paraffin-embedded tumor tissues. Anal Biochem 391: 166-168, 2009.

16. Tsiatis AC, Norris-Kirby A, Rich RG, et al: Comparison of Sanger sequencing, pyrosequencing and melting curve analysis for the detection of KRAS mutations. J Mol Diagn 12: 425-432, 2010.

17. Kim SK, Kim DL, Han HS, et al: Pyrosequencing analysis for detection of a BRAFV600E mutation in an FNAB specimen of thyroid nodules. Diagn Mol Pathol 17: 118-125, 2008.

18. Inoue A, Kobayashi K, Usui K, et al: First-line gefitinib for patients with advanced non-small cell lung cancer harboring epidermal growth factor receptor mutations without indication for chemotherapy. J Clin Oncol 27: 1394-1400, 2009.

19. Maemondo M, Inoue A, Kobayashi K, et al: Gefitinib or chemotherapy for non-small-cell lung cancer with mutated EGFR. N Engl J Med 362: 2380-2388, 2010.

20. Ettinger DS, Akerley W, Bepler G, et al: Non-small cell lung cancer. J Natl Compr Canc Netw 8: 740-801, 2010.

21. Pirker R, Herth FJ, Kerr KM, et al: Consensus for EGFR mutation testing in non-small cell lung cancer: results from a European workshop. J Thorac Oncol 5: 1706-1713, 2010.

22. Ronaghi M: Pyrosequencing sheds light on DNA sequencing. Genome Res 11: 3-11, 2001.

23. Ogino S, Kawasaki T, Brahmandam M, et al: Sensitive sequencing method for KRAS mutation detection by pyrosequencing. J Mol Diagn 7: 413-421, 2005.

24. Uhlmann K, Brinckmann A, Toliat MR, et al: Evaluation of a potential epigenetic biomarker by quantitative methylsingle nucleotide polymorphism analysis. Electrophoresis 23: 4072-4079, 2002.

25. Tost J, Dunker J and Gut IG: Analysis and quantification of multiple methylation variable positions in $\mathrm{CpG}$ islands by pyrosequencing. Biotechniques 35: 152-156, 2003.

26. Forbes SA, Bharma G, Bamford S, et al: The catalogue of somatic mutations in cancer (COSMIC). Curr Protoc Hum Genet Chapter 10: unit 10.11, 2008.

27. Rekhtman N, Brandt SM, Sigel CS, et al: Suitability of thoracic cytology for new therapeutic paradigms in non-small cell lung carcinoma: High accuracy of tumor subtyping and feasibility of EGR and KRAS molecular testing. J Thor Oncol 6: 451-458, 2011

28. Tokumo M, Toyooka S, Kiura K, et al: The relationship between epidermal growth factor receptor mutations and clinicopathologic features in non-small cell lung cancers. Clin Cancer Res 11: 1167-1173, 2005 .

29. Shigematsu H, Lim L, Takahashi T, et al: Clinical and biological features associated with epidermal growth factor receptor gene mutations in lung cancers. J Natl Cancer Inst 97: 339-346, 2005. 